\title{
Vibroacoustical Damping Diagnostics: Complex Frequency Response Function versus its Magnitude
}

\author{
L. Gelman ${ }^{\dagger}$, P. Jenkin and I. Petrunin \\ Department of Process and Systems Engineering, Cranfield University, Cranfield, MK 43 OAL, UK
}

M. J. Crocker ${ }^{\dagger}$
Department of Mechanical Engineering, Auburn University, Auburn, AL, 36849, USA

(Received 22 September 2004; accepted 17 May 2006)

In this paper new generic approach is proposed for damping diagnostics based on a forced oscillation method with sinusoidal and random white noise excitation. The main contribution of the paper is the development of a novel diagnostic technique. The proposed new approach and the traditional magnitude approach are compared and it is shown that the proposed approach provides an essential effectiveness gain over that of the magnitude approach.

${ }^{\dagger}$ Member of the International Institute of Acoustics and Vibration (IIAV)

\section{INTRODUCTION}

A new generic approach is proposed and generalised for those cases in which Fourier transforms are used for pattern recognition and diagnostics. ${ }^{1-3}$ The new approach consists of simultaneously using the real and imaginary components of the Fourier transform as pattern recognition (diagnostic) features and taking into account the covariance between these features. Theoretical insight related to this approach is described in references. ${ }^{1,3}$

This approach differs from most published applications for pattern recognition and diagnostics, in which the power spectral density (i.e. magnitude) or phase spectrum is used. In the present paper, some applications of the proposed generalised approach to damping diagnostics are considered.

Numerous techniques have been used to diagnose the damping ratio of objects. Of these techniques, the forced resonant technique and the impulse technique appear to be the most widely accepted. ${ }^{4}$ Both techniques are based on the frequency response function (FRF). Changes in damping will modify the parameters of the FRF; because if a parameter (e.g. stiffness, damping, etc.) of a physical system changes, then the FRF will be expected to change. ${ }^{4}$

Without loss of generality one can consider the damping diagnostics based on the resonance technique and two types of excitation: the sine excitation with random initial phase and white noise excitation. ${ }^{5}$ Diagnosis with the sine excitation is the most traditional and widely used approach for linear systems. ${ }^{5}$ White noise excitation is widely used for systems with multiple vibration modes. All resonance frequencies are excited simultaneously using this type of excitation.

The main purposes of this brief contribution are the

- development and validation of the novel vibroacoustical approach for damping monitoring based on the proposed generic approach, which essentially improves the monitoring accuracy:-3
- comparison of the results from the new approach with those obtained from the traditional approach based on the FRF magnitude.

\section{THE PROPOSED APPROACH}

The non-dimensional equation of motion of the linear oscillator, the system investigated for sinusoidal excitation, is described by the following expression:

$$
\ddot{X}+2 h \dot{X}+\omega_{n}^{2} X=\sin \left(\omega_{f} t+\varphi\right),
$$

where $X(t)=x(t) m / F$ is the normalised displacement; $x(t)$ is the displacement; $h=c / 2 m$ and $\omega_{n}=\sqrt{k / m_{n}}$ is the circular natural frequency; $m, c$ and $k$ are the object mass, damping, and stiffness, respectively; $F, \omega_{f}$ and $\varphi$ are the constant excitation amplitude, angular forcing frequency, and random initial phase, respectively.

For convenience, normalisation of the displacement, which makes the equation of motion invariant of excitation amplitude is used. It is possible to use this normalisation because the excitation amplitude is constant.

The random initial phase is uniformly distributed in the interval $[0,2 \pi]$. Forced resonant oscillations are used, and therefore, the excitation frequency is equal to the natural frequency.

The system motion undergoing the white noise excitation is also considered. The non-dimensional equation of motion under the Gaussian white noise excitation is described by the following equation:

$$
\ddot{x}+2 \zeta \omega_{n} \dot{x}+\omega_{n}^{2} x=A_{e}(t) \cos \varphi(t),
$$

where $A_{e}(t)=A_{i}(t) / m, A_{e}(t)$ and $A_{i}(t)$ are the normalised and un-normalised random Rayleigh envelopes of the Gaussian white noise excitation; $\varphi(t)$ is the random phase, and $\zeta=c /(2 \sqrt{\mathrm{km}})]$ is the damping ratio. 